\title{
Hyperhomocysteinemia is an independent risk factor of atherosclerosis in patients with metabolic syndrome
}

\author{
Giuseppina Piazzolla* $\mathbb{0}$, Mafalda Candigliota, Margherita Fanelli, Anna Castrovilli, Elsa Berardi, \\ Gianfranco Antonica, Stefano Battaglia, Vincenzo Solfrizzi, Carlo Sabbà and Cosimo Tortorella
}

\begin{abstract}
Background: Metabolic syndrome (MetS) is a clinical condition potentially promoting the development of atherosclerotic disease. To date, the clinical impact of elevated serum homocysteine (Hcy) levels in MetS is still under discussion. The aim of this cross sectional study was to evaluate the relationship between MetS and hyperhomocysteinemia and the potential role of Hcy in the pathogenesis of atherosclerotic complications of MetS.

Methods: We recruited 300 outpatients with MetS. All patients underwent a medical history collection, physical examination, blood sampling and carotid ultrasound echo-color Doppler. According to Hcy levels, MetS patients were divided into two groups: "normal" ( $<10.7 \mu \mathrm{mol} / /$; $n=140$, group 1) and "high" Hcy ( $\geq 10.7 \mu \mathrm{mol} / \mathrm{l} ; \mathrm{n}=160$, group 2). Comparisons between groups were made by Student's t-test or Chi-square test. The effects of potential covariates on group differences were evaluated by general linear models. The relationships between continuous variables were assessed by simple or multiple correlation and by linear regression. Multiple regression models were built to evaluate the effects of Hcy, together with other potential risk factors, on carotid atherosclerosis.

Results: Patients with high Hcy were predominantly male and slightly older than group 1 patients. Smokers and nonsmokers exhibited similar Hcy levels, nor was a statistical relationship between pack-years and Hcy observed. Group 2 showed lower levels of folic acid, vitamin D, high density lipoprotein (HDL)-cholesterol and glomerular filtration rate (e-GFR) than group 1, but higher levels of C-peptide, uric acid and triglycerides. In all patients, Hcy was positively correlated with C-peptide and uric acid and negatively with folic acid and e-GFR. Intima-media thickness (IMT) and carotid stenosis degree were significantly higher in patients with high Hcy and a positive relationship between Hcy and both IMT and carotid stenosis was detected in all patients. Finally, Hcy atherogenic effects were independent of other well-known atherosclerosis risk factors.

Conclusions: Our results highlight a link between MetS and hyperhomocysteinemia and a direct effect of Hcy on atherogenic process during MetS. Early correction of folic acid levels may contribute to prevent cardiovascular complications in MetS patients.
\end{abstract}

Keywords: Homocysteine, Metabolic syndrome, IMT, Carotid stenosis, C-peptide, Folic acid, Pack-years

*Correspondence: giuseppina.piazzolla@uniba.it

Interdisciplinary Department of Medicine, University of Bari School

of Medicine, Piazza G. Cesare 11, 70124 Bari, Italy 


\section{Background}

Cardiovascular diseases are still the main cause of mortality worldwide. Early identification, prevention and treatment of the main cardiovascular risk (CVR) factors are undoubtedly priority goals to be achieved. In this context, the metabolic syndrome (MetS) and increased serum homocysteine (Hcy) levels have been described as independent risk factors for cardiovascular diseases [1, 2].

Epidemiological and clinical data support an important link between MetS and hyperhomocysteinemia, and some studies have even suggested that increased serum levels of this amino acid may be an additional constituent of MetS [3]. However, the exact nature of this relationship remains unclear. In all these clinical conditions the complex interaction of genetic and environmental factors, among which a sedentary lifestyle and overnutrition play a determining role, contributes to the onset and persistence of a low-grade systemic inflammation. This proinflammatory state, in turn, seems to affect the incidence of systemic complications, first of all atherosclerotic disease [4].

The MetS is a common condition whose prevalence is rising to epidemic proportions, having a significant impact on public health and the global economy [5, 6]. Far from being a classic disease in itself, it is rather defined as a cluster of metabolic disorders (i.e. insulin resistance, central obesity, dyslipidemia, hypertension, endothelial dysfunction) whose coexistence in the same individual predisposes to cardiovascular (CV) events, type-2 diabetes and nonalcoholic fatty liver disease [5].

Since 1998, scientific organizations have proposed several closely related but individual definitions of the syndrome, using diagnostic criteria based on different cut-off points of risk values for waist circumference, blood pressure and serum levels of glucose, triglycerides and high density lipoprotein (HDL)-cholesterol. Regardless of which cluster of criteria was adopted, the common purpose has been to make an early identification of highrisk patients and potential CV complications. In 2009, the so-called "harmonizing definition" of MetS assigned equivalent levels of importance to all of its components, thus reconciling all previous definitions [7].

Homocysteine is a dietary amino acid produced by the breakdown of methionine [8] whose metabolic pathway involves several enzymes. Circulating Hcy is, in fact, maintained at relatively low levels both by vitamin B12/ folate-dependent enzymatic conversion to methionine and via a trans-sulfuration pathway forming cysteine [9]. Genetic variability of one of these enzymatic activities $[10,11]$, as well as folate or vitamin B12 deficiencies [12], may induce increased levels of cellular and serum Hcy, considered to be toxic to cells [8]. Other conditions associated with hyperomocysteinemia include age, smoking and male sex.

The primary purpose of the study was to evaluate the controversial relationship between Hcy and Mets. At the same time, the possible role of Hcy in the pathogenesis of atherosclerotic complications of the MetS was investigated via color-doppler ultrasonography of carotid vessels, a common method that has proven to be both reliable and inexpensive.

\section{Patients and methods Study population}

In this cross sectional study, 300 consecutive patients with MetS (182 males and 118 females; mean age 63 years, range 26-86 years) attending the Metabolic Disorders Outpatients Clinic of the Department of Internal Medicine at the University Hospital of Bari, were enrolled from December 2017 to November 2018. MetS was diagnosed, according to the "harmonizing definition" of the syndrome [7], when at least three of the following criteria were present: (1) waist circumference $\geq 94 \mathrm{~cm}$ in European men or $\geq 80 \mathrm{~cm}$ in European women; (2) fasting glucose $>100 \mathrm{mg} / \mathrm{dl}$ or ongoing therapy for elevated glucose levels; (3) triglycerides $\geq 150 \mathrm{mg} /$ $\mathrm{dl}$ or specific treatment for this lipid abnormality; (4) $\mathrm{HDL}<40 \mathrm{mg} / \mathrm{dl}$ in men or $<50 \mathrm{mg} / \mathrm{dl}$ in women or specific treatment for this lipid abnormality; (5) systolic blood pressure $\geq 130 \mathrm{mmHg}$ and/or diastolic blood pressure $\geq 85 \mathrm{mmHg}$ or ongoing therapy for hypertension. Exclusion criteria were any kind of cancer within less than 5 years prior to the study, infections and systemic corticosteroid treatment within 4 weeks prior to the study. All patients underwent a general examination, including the following measures: height, weight, Body Mass Index (BMI), waist circumference, arterial pressure; venous sampling for routine analysis, including serum Hcy, uric acid, vitamin B12, folic acid, 25-OH vitamin D, $\mathrm{C}$-peptide and glycated haemoglobin (HbA1c), after 12-h fasting period; and instrumental tests including echocolor Doppler of the supra-aortic vessels.

Patients were subdivided into two groups according to the detection of "normal" $(<10.7 \mu \mathrm{M} /$ l; group 1$)$ or "high" ( $\geq 10.7 \mu \mathrm{M} / \mathrm{l}$; group 2 ) serum Hcy values.

All biochemical measurements were centralized and performed in the ISO 9001 certified laboratories of the University Hospital of Bari. Hcy and uric acid were determined by the URCA method on the Dimension Vista System (Siemens Healthcare Diagnostic Products GmbH, Marburg, Germany); vitamin B12 and folic acid were assessed by chemiluminescent immunoassay (CLIA) on the ADVIA Centaur System (Siemens); C-peptide and $25-\mathrm{OH}$ vitamin $\mathrm{D}$ were determined by CLIA on the LIAISON analyzer (DiaSorin Inc, Stillwater, MN, USA); 
HbA1c was assessed in human whole blood using ionexchange high-performance liquid chromatography (HPLC) on the Bio-Rad Variant II Hemoglobin A1c Program (BIO-RAD Laboratories Srl, Milan, Italy).

Echo-color Doppler, always carried out by the same two operators, allowed us to measure the Intima Media Thickness (IMT) and to calculate the degree of carotid stenosis. All the examinations were performed with high resolution B-mode ultrasonography (Esaote Mylab Twice) using a 7-12 MHz vascular transducer in multiple projections (longitudinal and transverse scans) to optimize detection of the parameters. The evaluation of the carotid arteries included three modalities: B-mode, in order to get images in grey scale for the measurement of IMT $1 \mathrm{~cm}$ proximal to the carotid bulb; color doppler and spectral analysis, in order to obtain the blood flow velocity. Combining all the information collected through these three different approaches it was possible to study the degree of stenosis and to classify its severity in categories according to ultrasound and doppler flowmetric values. In particular, the NASCET (North American Symptomatic Carotid Endarterectomy Trial) method was used to quantify the degree of stenosis. The IMT, defined as the distance between the intimal-luminal interface and the medial-adventitial interface, was considered pathological if greater than $0.9 \mathrm{~mm}$.

The HOMA (Homeostasis Model Assessment) Index was calculated as (fasting insulin $\times$ fasting glucose) $/ 405$. A pack-year was defined as 20 cigarettes smoked every day for 1 year.

The study was approved by the Clinical Investigation Ethics Committee of the University of Bari Medical Center (Ethical approval number: PZZ/SM_BPCO/2017), and all patients gave written informed consent to take part.

\section{Statistical analysis}

Comparisons between groups were made by Student's t-test and Chi-square test for continuous and categorical variables, respectively. As age, gender and smoking habit were significantly different between patients with normal or high Hcy, the analysis of covariance was carried out by general linear models to correct further differences between the two groups by the effects of these covariates on Hcy.

The relationship between independent and dependent continuous variables was assessed by linear regression. The effects of Hcy, together with age, pack-years, sex and other potential atherosclerosis risk factors, on IMT and carotid stenosis were evaluated by multiple regression models. In particular, a forward stepwise selection based on Akaike information criterion (AIC) was performed after an automatic smoothing of outliers and a correction of missing values.

Any other association among variables was evaluated by simple or multiple correlation. In the latter context, partial Pearson correlation coefficients were calculated for each pair of variables when the effect of remaining variables had been removed.

Statistical analysis was performed with SPSS version 23.

\section{Results}

The baseline characteristics of our study population are summarized in Table 1 . More than $50 \%$ of total patients exhibited higher than normal levels of serum Hcy.

The subset of patients with high Hcy (group 2) consisted mainly of males. This is no coincidence, as the amino acid levels appeared to be significantly higher in males than in females (mean \pm SD: $12.7 \pm 4.7$ vs $10.5 \pm 3.9$, respectively; $\mathrm{t}=3.780, \mathrm{p}<0.001)$. In addition, the patients in group 2 were slightly but significantly older than those in group 1, in line with known epidemiological data. The number of pack-years was higher in patients with high Hcy, and the percentage of smokers was significantly different between the two groups (Table 1). Nevertheless, Hcy levels were not different between smoker and non smoker patients (Fig. 1a) and no relationship was detected in the whole population between pack-years and serum Hcy levels (Fig. 1b).

Given the role of sex, age and smoking on Hcy metabolism, these variables were used as covariates to correct the additional differences detected between patients with normal or high Hcy by their potential effects. Accordingly, for all other continuous variables, corrected means, as estimated by the analysis of covariance model, are reported in Table 1.

All patients showed high absolute values of waist circumference, BMI and HOMA Index but no differences between the two groups were observed as regards these or other clinical variables (Table 1). As concerns the blood chemistry data, the group of patients with high Hcy was characterized by higher values of C-peptide, triglycerides and uric acid and lower concentrations of HDL-cholesterol, folates and vitamin D compared to the other group (Table 1). Interestingly, half of these variables appeared to be related to serum Hcy. A multiple correlation analysis between Hcy and all these variables, in fact, revealed a negative correlation between Hcy and folate as well as a positive association between Hcy and C-peptide or uric acid (Table 2).

The analysis of kidney function tests highlighted higher levels of (blood urea nitrogen) BUN and creatinine, and lower values of glomerular filtration rate (e-GFR) in the subset of patients with high Hcy 
Table 1 Baseline characteristics of the total study population

\begin{tabular}{|c|c|c|c|c|}
\hline & $\begin{array}{l}\text { Patients with normal } \\
\text { homocysteine (group 1; } \\
n=140 \text { ) }\end{array}$ & $\begin{array}{l}\text { Patients with high } \\
\text { homocysteine (group 2; } \\
n=160 \text { ) }\end{array}$ & Statistical analysis & Significance (p) \\
\hline Age (years) & $61.4 \pm 0.9$ & $64.1 \pm 1.0$ & $t=-2.015$ & $p=0.045$ \\
\hline Gender, male [n (\%)] & $67(47.9 \%)$ & $115(71.9 \%)$ & $x^{2}=18.050$ & $p<0.001$ \\
\hline Smokers [n (\%)] & $58 / 137(42.3 \%)$ & $85 / 155(54.8 \%)$ & $x^{2}=4.549$ & $p=0.033$ \\
\hline Never smokers [n (\%)] & 79/137 (57.7\%) & $70 / 155$ (45.2\%) & & \\
\hline $\begin{array}{l}\text { Pack-years (n) } \\
\text { a }\end{array}$ & $11.6 \pm 1.9$ & $19.4 \pm 2.5$ & $t=-2.464$ & $p=0.014$ \\
\hline Waist circumference $(\mathrm{cm})$ & $104.1 \pm 1.1$ & $105.3 \pm 1.1$ & $F=0.62$ & $p=0.432$ \\
\hline $\mathrm{BMI}\left(\mathrm{kg} / \mathrm{m}^{2}\right)$ & $29.7 \pm 0.5$ & $30.0 \pm 0.5$ & $F=0.20$ & $p=0.659$ \\
\hline $\mathrm{SBP}(\mathrm{mmHg})$ & $129.2 \pm 1.2$ & $130.9 \pm 1.2$ & $F=0.96$ & $p=0.328$ \\
\hline $\mathrm{DBP}(\mathrm{mmHg})$ & $79.3 \pm 0.8$ & $79.0 \pm 0.8$ & $F=0.04$ & $p=0.837$ \\
\hline \multicolumn{5}{|l|}{ MetS criteria (n) } \\
\hline 3 & $60(42.9 \%)$ & $60(37.5 \%)$ & $x^{2}=6.723$ & $p=0.035$ \\
\hline 4 & $63(45.0 \%)$ & $62(38.7 \%)$ & & \\
\hline 5 & $17(12.1 \%)$ & $38(23.8 \%)$ & & \\
\hline Fasting glucose (mg/dl) & $113.0 \pm 3.4$ & $116.1 \pm 3.4$ & $F=0.41$ & $p=0.521$ \\
\hline $\mathrm{HbA} 1 \mathrm{c}(\mathrm{mmol} / \mathrm{mol})$ & $45.6 \pm 1.2$ & $43.9 \pm 1.2$ & $F=0.97$ & $p=0.324$ \\
\hline C-peptide (ng/ml) & $2.0 \pm 0.1$ & $2.4 \pm 0.1$ & $F=7.10$ & $p=0.008$ \\
\hline Insulin (microUl/ml) & $11.4 \pm 0.7$ & $12.0 \pm 0.7$ & $F=0.30$ & $p=0.587$ \\
\hline HOMA Index & $3.1 \pm 0.2$ & $3.4 \pm 0.2$ & $F=0.79$ & $p=0.376$ \\
\hline Vitamin D (ng/ml) & $23.5 \pm 0.9$ & $21.0 \pm 0.9$ & $F=4.06$ & $p=0.045$ \\
\hline Folic acid (ng/ml) & $9.0 \pm 0.4$ & $6.7 \pm 0.4$ & $F=15.76$ & $p<0.001$ \\
\hline Vitamin B12 (pg/ml) & $414.6 \pm 29.6$ & $401.0 \pm 28.8$ & $F=0.11$ & $p=0.744$ \\
\hline e-GFR (ml/min) & $90.4 \pm 1.2$ & $81.3 \pm 1.2$ & $F=26.85$ & $p<0.001$ \\
\hline Creatinine (mg/dl) & $0.78 \pm 0.03$ & $0.93 \pm 0.03$ & $F=15.27$ & $p<0.001$ \\
\hline BUN (mg/dl) & $37.0 \pm 1.2$ & $44.8 \pm 1.2$ & $F=19.11$ & $p<0.001$ \\
\hline Uric acid (mg/dl) & $4.5 \pm 0.1$ & $5.0 \pm 0.1$ & $F=12.32$ & $p=0.001$ \\
\hline Cholesterol (mg/dl) & $163.4 \pm 3.1$ & $165.0 \pm 3.1$ & $F=0.14$ & $p=0.705$ \\
\hline LDL-cholesterol (mg/dl) & $85.9 \pm 2.9$ & $87.7 \pm 2.9$ & $F=0.19$ & $p=0.662$ \\
\hline HDL-cholesterol (mg/dl) & $53.6 \pm 1.1$ & $49.2 \pm 1.1$ & $F=4.10$ & $p=0.044$ \\
\hline Triglycerides (mg/dl) & $122.1 \pm 6.0$ & $148.8 \pm 5.9$ & $F=9.50$ & $p=0.002$ \\
\hline Carotid atherosclerosis $^{\mathrm{b}}[\mathrm{n}(\%)]$ & $111 / 133(83.5 \%)$ & 139/154 (90.3\%) & $x^{2}=2.939$ & $p=0.086$ \\
\hline Carotid stenosis (\%) & $19.1 \pm 1.6$ & $23.5 \pm 1.4$ & $F=4.19$ & $p=0.042$ \\
\hline $\mathrm{IMT}(\mathrm{mm})$ & $1.02 \pm 0.01$ & $1.10 \pm 0.02$ & $F=4.04$ & $p=0.046$ \\
\hline
\end{tabular}

Data are presented as mean \pm SE or as frequency and percentage. Evaluation of group differences was done with Student's $t$ test and the Chi-square test for continuous and categorical variables, respectively

A pack-year is defined as 20 cigarettes smoked every day for 1 year

$B M I$ Body Mass Index, SBP systolic blood pressure, DBP diastolic blood pressure, HbA1c glycated haemoglobin, HOMA (Homeostasis Model Assessment) Index: fasting glucose $\times$ fasting insulin/405, e-GFR glomerular filtration rate, BUN blood urea nitrogen, $L D L$ low density lipoprotein, $H D L$ high density lipoprotein, IMT Intima Media Thickness

a Age, sex and smoking were used as covariates to perform further evaluations by general linear models. Therefore, from here, continuous variable values are expressed as corrected means, as estimated by the model

b Patients showing an IMT greater than $0.9 \mathrm{~mm}$ and/or a carotid stenosis

(Table 1). In addition, examining MetS individuals as a whole, a negative correlation between Hcy and e-GFR $(\mathrm{r}=-0.441 ; \mathrm{p}<0.001)$ and, concordantly, a positive correlation of the amino acid concentration with both BUN $(r=0.557 ; \mathrm{p}<0.001)$ and creatinine $(\mathrm{r}=0.624$; $\mathrm{p}<0.001$ ), were observed.
Finally, we evaluated the possible relationship between Hcy and atherosclerosis in the course of MetS. In this respect, it should be outlined that more than $80 \%$ of our patients showed signs of carotid atherosclerosis, defined as an IMT greater than $0.9 \mathrm{~mm}$ and/or a carotid stenosis. This finding was independent of the levels of Hcy, 


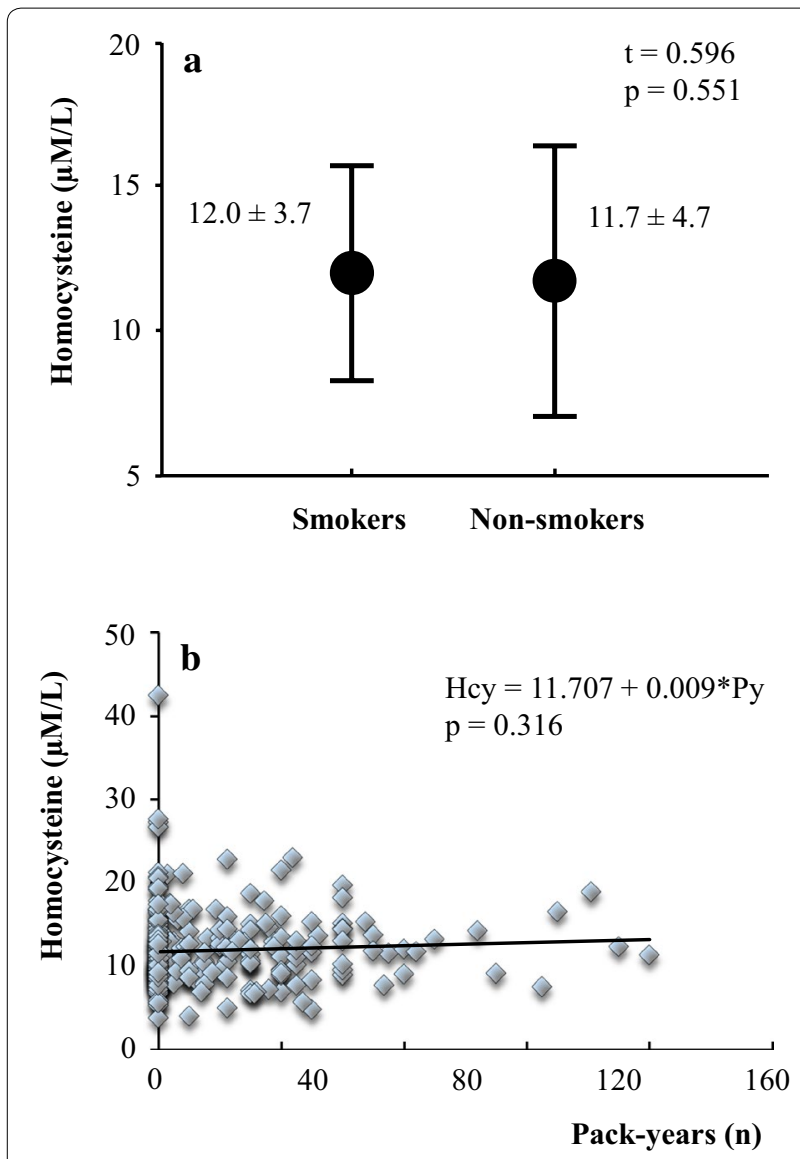

Fig. 1 a Hcy levels in smoker and non-smoker patients with MetS. Values are expressed as mean \pm SD. $\mathbf{b}$ Relation between pack-years and Hcy in all MetS patients. Each symbol identifies a single individual

the percentage of patients with ultrasound signs of atherosclerosis being not significantly different in the subset with high compared with normal amino acid serum concentrations (Table 1). However, when the degree of atherosclerosis was quantified, in terms of either IMT or carotid stenosis, values were significantly greater in group 2 (Table 1). Consistent with these data, a significant effect of Hcy levels on IMT or carotid stenosis degree was detected in the whole population of MetS patients, as indicated by the positive relation between Hcy and either of these atherosclerosis clinical signs (Fig. 2a). Accordingly, we deemed it necessary to assess whether the promoting action of Hcy on atherosclerotic disease might be due to other confounding atherosclerosis risk factors, including, in particular, a smoking habit.
This did not seem to be the case as a significant positive relation between Hcy and IMT or carotid stenosis was observed even when considering only non smoker MetS patients (Fig. 2b).

Furthermore, multiple regression analysis demonstrated that Hcy, pack-years and age, but not sex, systolic blood pressure, e-GFR, low density lipoprotein (LDL)cholesterol or triglycerides, were able to affect IMT (Final Model: $\mathrm{IMT}=0.466+0.07 *$ Age $+0.02 * \mathrm{Py}+0.008 * \mathrm{Hcy}$; $\mathrm{p}<0.001)$. Similar results were detected when the carotid stenosis degree was evaluated (Final Model: Carotid stenosis $=-24.554+0.595 *$ Age $+0.22 * \mathrm{Py}+0.811 *$ Hcy; $\mathrm{p}<0.001$ ).

The analysis of the atherogenic effect of uric acid was not among the purposes of the study. Nevertheless, the observation that uricemia did not relate either with IMT (IMT $=1.04+0.005^{*}$ Uric acid; $\mathrm{p}=0.614$ ) or with the percentage of carotid stenosis (Carotid stenosis $=15.75+1.22 *$ Uric acid; $\mathrm{p}=0.173)$, although incidental, is worthy of mention.

\section{Discussion}

An increasing number of studies over the last 20 years has underlined the role of Hcy as a potential risk factor for $\mathrm{CV}$ diseases, venous thrombosis, vascular complications on atherosclerotic basis, systemic disorders, cognitive decline [1, 13-17]. It has also been reported that its effects on atherogenic processes are particularly relevant in diabetic patients [18-20]. To date, despite the ample volume of literature available, the clinical impact of elevated serum Hcy levels is still not clear, and whether Hcy is a mediator, a biomarker or just an epiphenomenon of cardiovascular and/or neurological diseases is still under discussion [9].

In this context, the MetS, a clinical condition associated with a high mortality for $\mathrm{CV}$ events and for all causes, appears to be closely linked to homocysteinemia but the expert opinions on the exact nature of this relationship are controversial [21, 22]. In fact, the data provided by studies in both the general population and specific subgroups of individuals, such as adolescents, obese patients, or those with previous CV disease [19, 21, $23,24]$, are conflicting.

The present study outlines a significant association between the MetS and hyperhomocysteinemia. Contrary to the prevalence of hyperhomocysteinemia ranging around $5-10 \%$ in the adult general population, with

Table 2 Partial correlation coefficients between Hcy and metabolic variables in MetS patients

\begin{tabular}{lllllll}
\hline & C-peptide & Triglycerides & Uric acid & HDL-cholesterol & Folic acid & Vitamin D \\
\hline Homocysteine & $r=0.274$ & $r=-0.047$ & $r=0.182$ & $r=-0.410$ & $r=-0.287$ & $r=-0.050$ \\
& $p<0.001$ & $p=0.483$ & $p=0.006$ & $p=0.540$ & $p<0.001$ & $p=0.452$ \\
\hline
\end{tabular}


a All MetS patients

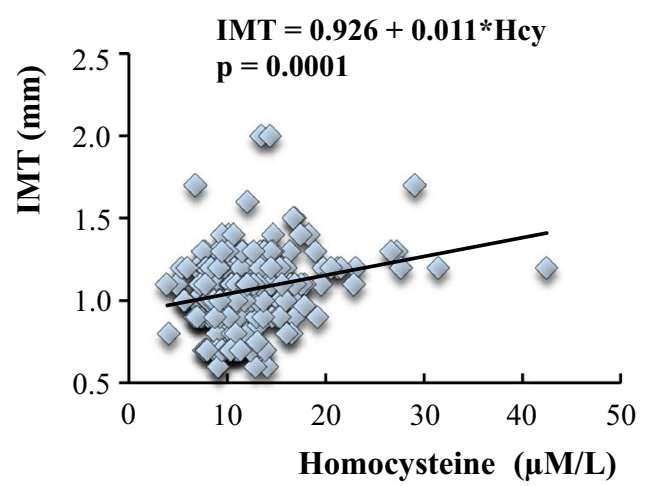

\section{b Non smoker MetS patients}

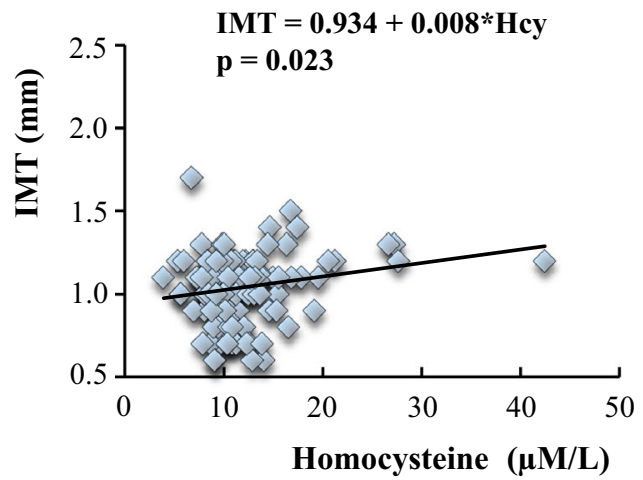

Carotid stenosis $=8.748+1.073 * \mathbf{H c y}$

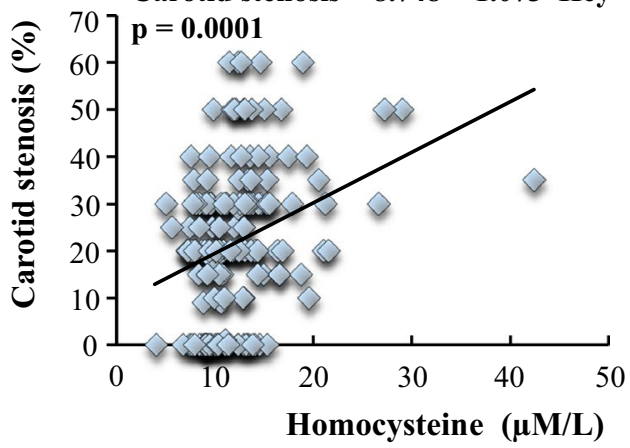

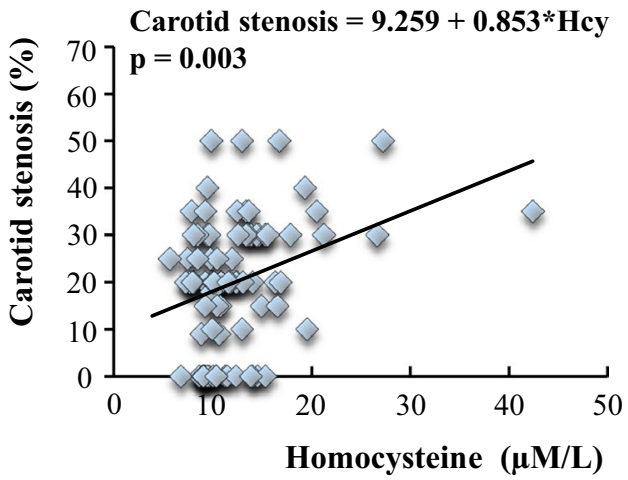

Fig. 2 Effects of Hcy on IMT and carotid stenosis degree in all (a) and in non smoker (b) MetS patients. Each symbol identifies a single individual

peaks of $30 \%$ in the elderly [25], a very high percentage of MetS patients included in the study (53.3\%) showed higher than normal Hcy levels. In line with the epidemiological data for the general population, a large proportion of these patients was male (71.9\%), likely as a result of the gender-dependent production of Hcy being higher in males than females [26]. The comparison of MetS patients with normal or elevated serum Hcy levels highlighted the fact that the group with high Hcy exhibited significantly higher triglycerides and lower HDL-cholesterol values, two parameters that contribute directly to the diagnosis of MetS. Interestingly, the same subset of patients also showed significantly higher levels of uric acid, a molecule that, like Hcy, is counted among the emerging CVR factors, a serum increase being considered a potential additional diagnostic criterion of the MetS.

A particularly interesting point emerged from the analysis of the smoking habit variable, as cigarette smoke is described to induce both insulin resistance [27] and Hcy production [8]. The group with high Hcy displayed higher values of pack-years and a greater percentage of smoking individuals (including both ex-smokers and current smokers) as compared with the other subset. Nevertheless, such an important CVR factor did not appear to affect the amino acid serum levels in patients with MetS, as serum Hcy was similar in smokers and non-smokers and, above all, no significant relation between pack-years and Hcy levels was observed in the overall study population. According to these data, we conclude that a smoking habit is not a discriminating factor between MetS patients with high or normal homocysteinemia and, therefore, it should not significantly influence Hcy-mediated cardiovascular effects in our patients.

In this regard, it is worth noting that MetS patients with high Hcy exhibited significantly greater C-peptide values compared with the other group. As known, C-peptide levels depend only on insulin production, are not affected by insulin replacement and are directly related to insulin resistance which, in turn, plays a pivotal role in the pathogenesis of MetS. Accordingly, the evidence of a positive correlation between Hcy and 
C-peptide in the whole study population makes it reasonable to suppose that different concentrations of circulating Hcy could account for the different degree of insulin resistance among patients with MetS. Although scientific reports on this topic are not conclusive, our results are in line with previous observations showing that high serum levels of Hcy are associated with an increased risk to develop Type II diabetes mellitus, are closely associated to $\beta$-cell dysfunction and insulin resistance via oxidative- and inflammation-mediated pathways $[28,29]$ and may contribute to cause diabetes-related complications such as endothelial and cardiovascular damage [19, 30], retinopathy and nephropathy [20,30-34]. The potential capacity of Hcy to mediate kidney damage is consistent with our findings of significant differences between the two groups of MetS patients with reference to the main renal function test parameters. This view is confirmed by the negative correlation between Hcy and e-GFR as well as by the positive correlation of Hcy with both BUN and creatinine noted in all the patients included in the study. However, renal function of MetS patients with high Hcy appeared to be preserved, suggesting that Hcy should rather be considered as an early marker of renal impairment in MetS, similarly to what was recently suggested [35].

As already described in the general population, we confirmed in patients with MetS the negative relationship between homocysteinemia and folic acid. Considering the key role of folates in the metabolism of methionine [12] and our observation of a significant reduction of folate concentrations in MetS patients with high Hcy, we suggest that any folic acid deficiency occurring in these patients be promptly corrected. Such a therapy would, in fact, improve this methionine metabolic disorder thought to contribute to the CV complications of the MetS, without any significant side effect. Vitamins B supplementation is undoubtedly effective in lowering serum Hcy levels, but data on its effects on CVR reduction are not conclusive. Evidence has been provided that Hcy-lowering vitamin $\mathrm{B}$ treatments failed to improve $\mathrm{CV}$ outcomes and to reduce death from any cause or stroke recurrence in patients with or without previous ischemic stroke or known CV disease, and this would not seem to recommend the systematic use of such therapeutic regimens in patients with a CV disease history $[25,36]$. Conversely, it has also been reported that supplementation with folic acid, alone or in combination with vitamin B6 and B12, is effective in reducing stroke risk in patients with known CV disease [25, 37, 38] and, above all, can significantly decrease the risk for first stroke in hypertensive adults without a CV disease history [39-42]. This particular capacity of Hcy-lowering therapy to provide an effective primary prevention against $\mathrm{CV}$ events strengthens our proposal to systematically treat folic acid deficiency in MetS patients. Further support of this approach is provided by the outcomes of a recent systematic review and meta-analysis of several randomized controlled trials addressing the effects of metformin, a first-line insulinsensitizing drug, on Hcy serum levels [43]. Authors concluded that, although metformin does not directly affect Hcy levels, it would induce an increase in the amino acid concentration when patients are not appropriately supplemented with group B vitamins and/or folates. This effect is considered evidently detrimental by the American Association of Clinical Endocrinologists, whose guidelines do, in fact, suggest that vitamin B12/folates be added in patients under treatment with metformin [44].

It cannot be denied that the real significance of high circulating Hcy has not yet been fully elucidated. To gain insight into this issue, we investigated the atherogenic role of Hcy in our insulin resistant patients. In this context, it should be emphasized that the group with high Hcy exhibited higher values of both IMT and carotid stenosis than the group with normal Hcy. Furthermore, in all patients with MetS, the increased Hcy concentration was paralleled by an analogous increase in IMT or carotid stenosis values. These findings are even more impressive considering that Hcy effects were not mediated by the smoking habit variable, being significant in non-smokers patients as well, nor by other well-known and potentially confounding atherosclerosis risk factors.

This allows us to speculate that Hcy may exert a direct role in the pathogenesis of vascular complications of MetS. Another intriguing aspect is that, even if uric acid shares with Hcy the ability to induce atherosclerosisassociated events (i.e. oxidative damage, endothelial cell dysfunction and smooth muscle cell proliferation), in our patients it did not exhibit any significant relationship with IMT or carotid stenosis, thus questioning its clinical role in the development of atherosclerotic process in the course of MetS.

Overall, the study has some potential limitations: (i) the number of patients would need to be increased in order to allow for some definitive conclusions to be drawn; (ii) although no patient used nutritional supplements, particular eating habits, potentially interfering with Hcy levels, were not directly evaluated.

Beyond these limitations, our results outline a strict relationship among insulin resistance, hyperhomocysteinemia and MetS, whose clinical and pathogenic interactions warrant further investigation. In addition, the enhancing role of Hcy on the progression of atherosclerotic process suggests that a correction of folate deficiency is advisable in order to prevent the $\mathrm{CV}$ morbidity and mortality that is so common in insulin resistant MetS patients. 


\begin{abstract}
Abbreviations
MetS: metabolic syndrome; Hcy: homocysteine; IMT: Intima Media Thickness; CV: cardiovascular; CVR: cardiovascular risk; HDL: high density lipoprotein; LDL: low density lipoprotein; BMI: Body Mass Index; HbA1c: glycated haemoglobin; NASCET: North American Symptomatic Carotid Endarterectomy Trial; HOMA: Homeostasis Model Assessment; SD: standard deviation; SE: standard error; e-GFR: glomerular filtration rate; BUN: blood urea nitrogen; SBP: systolic blood pressure.
\end{abstract}

\section{Acknowledgements}

We are grateful to Mary Pragnell for language revision.

\section{Authors' contributions}

GP: conception and design of the work; interpretation of data; drafting of the work. MC, AC, SB: acquisition of data. MF: statistical analysis of data. EB, GA: Echo-color Doppler operator. VS: interpretation of data. CS: revision of the work. CT: conception and design of the work; interpretation of data; revision of the work. All authors read and approved the final manuscript.

\section{Funding}

This work was supported by Grants from Università di Bari, Bari, Italy (ex 60\%).

\section{Availability of data and materials}

The datasets used during the current study are available from the corresponding author on reasonable request.

\section{Ethics approval and consent to participate}

The study was approved by the Clinical Investigation Ethics Committee of the University of Bari Medical Center (Ethical approval number: PZZ/SM_ $\mathrm{BPCO} / 2017)$, and all patients gave written informed consent to take part.

\section{Consent for publication}

Not applicable.

\section{Competing interests}

The authors declare that they have no competing interests.

Received: 9 Auqust 2019 Accepted: 15 October 2019

Published online: 26 October 2019

\section{References}

1. Wald DS, Law M, Morris JK. Homocysteine and cardiovascular disease: evidence on causality from a meta-analysis. BMJ. 2002;325:1202-6.

2. Catena C, Colussi G, Nait F, Capobianco F, Sechi LA. Elevated homocysteine levels are associated with the metabolic syndrome and cardiovascular events in hypertensive patients. Am J Hypertens. 2015;28:943-50.

3. Oron-Herman M, Rosenthal T, Sela BA. Hyperhomocysteinemia as a component of syndrome X. Metabolism. 2003;52:1491-5.

4. Taleb S. Inflammation in atherosclerosis. Arch Cardiovasc Dis. 2016;109:708-15.

5. Kastorini CM, Panagiotakos DB, Georgousopoulou EN, Laskaris A, Skourlis N, Zana A, ATTICA Study Group, et al. Metabolic syndrome and 10-year cardiovascular disease incidence: the ATTICA study. Nutr Metab Cardiovasc Dis. 2016;26:223-31.

6. Grundy SM. Metabolic syndrome pandemic. Arterioscler Thromb Vasc Biol. 2008;28:629-36.

7. Alberti KG, Eckel RH, Grundy SM, Zimmet PZ, Cleeman JI, Donato KA, International Diabetes Federation Task Force on Epidemiology and Prevention, National Heart, Lung, and Blood Institute, American Heart Association, World Heart Federation, International Atherosclerosis Society, International Association for the Study of Obesity, et al. Harmonizing the metabolic syndrome: a joint interim statement of the International Diabetes Federation Task Force on Epidemiology and Prevention; National Heart, Lung, and Blood Institute; American Heart Association; World Heart Federation; International Atherosclerosis Society; and International Association for the Study of Obesity. Circulation. 2009;120:1640-5.

8. Škovierová H, Vidomanová E, Mahmood S, Sopková J, Drgová A, Červeňová T, et al. The molecular and cellular effect of homocysteine metabolism imbalance on human health. Int J Mol Sci. 2016;17:1733.
9. Hannibal L, Blom HJ. Homocysteine and disease: causal associations or epiphenomenons? Mol Aspects Med. 2017;53:36-42.

10. Vaughn JD, Bailey LB, Shelnutt KP, Dunwoody KM, Maneval DR, Davis $\mathrm{SR}$, et al. Methionine synthase reductase $66 \mathrm{~A} \rightarrow \mathrm{G}$ polymorphism is associated with increased plasma homocysteine concentration when combined with the homozygous methylenetetrahydrofolate reductase 677C $\rightarrow$ T variant. J Nutr. 2004;134:2985-90.

11. Jhee $\mathrm{KH}$, Kruger WD. The role of cystathionine beta-synthase in homocysteine metabolism. Antioxid Redox Signal. 2005;7:813-22.

12. Ubbink JB, Vermaak WJ, van der Merwe A, Becker PJ. Vitamin B-12, vitamin B-6, and folate nutritional status in men with hyperhomocysteinemia. Am J Clin Nutr. 1993;57:47-53.

13. Homocysteine Studies Collaboration. Homocysteine and risk of ischemic heart disease and stroke: a meta-analysis. JAMA. 2002;288:2015-22.

14. Durand P, Prost M, Loreau N, Lussier-Cacan S, Blache D. Impaired homocysteine metabolism and atherotrombotic disease. Lab Invest. 2001;81:645-72.

15. Katsiki N, Perez-Martinez P, Mikhailidis DM. Homocysteine and noncardiac vascular disease. Curr Pharm Des. 2017;23:3224-32.

16. Smith DA, Refsum H. Homocysteine, B vitamins and cognitive impairment. Annu Rev Nutr. 2016;36:211-39.

17. Moretti R, Caruso P, Dal Ben M, Conti C, Gazzin S, Tiribelli C. Vitamin D, homocysteine and folate in subcortical vascular dementia and Alzheimer dementia. Front Aging Neurosci. 2017;9:169.

18. Kundi H, Kiziltunc E, Ates I, Cetin M, Barca AN, Ozkayar N, et al. Association between plasma homocysteine levels and end-organ damage in newly diagnosed type 2 diabetes mellitus patients. Endocr Res. 2017:42:36-41.

19. Soinio M, Marniemi J, Laakso M, Lehto S, Rönnemaa T. Elevated plasma homocysteine level is an independent predictor of coronary heart disease events in patients with type 2 diabetes mellitus. Ann Int Med. 2004;140:94-100.

20. Looker HC, Fagot-Campagna A, Gunter EW, Pfeiffer CM, Narayan KM, Knowler WC, et al. Homocysteine as a risk factor for nephropathy and retinopathy in type 2 diabetes. Diabetologia. 2003;46:766-72.

21. Sreckovic B, Sreckovic VD, Soldatovic I, Colak E, Sumarac-Dumanovic M, Janeski H, et al. Homocysteine is a marker for metabolic syndrome and atherosclerosis. Diabetes Metab Syndr. 2017;11:179-82.

22. Hajer GR, van der GraafY, Olijhoek JK, Verhaar MC, Visseren FL, SMART Study Group. Levels of homocysteine are increased in metabolic syndrome patients but are not associated with an increased cardiovascular risk, in contrast to patients without the metabolic syndrome. Heart. 2007:93:216-20.

23. Yakub M, Schulze KJ, Khatry SK, Stewart CP, Christian P, West KP. High plasma homocysteine increases risk of metabolic syndrome in 6 to 8 year old children in rural Nepal. Nutrients. 2014;6:1649-61.

24. Vayá A, Rivera L, Hernández-Mijares A, de la Fuente M, Solá E, Romagnoli $M$, et al. Homocysteine levels in morbidly obese patients: its association with waist circumference and insulin resistance. Clin Hemorheol Microcirc. 2012;52:49-56.

25. Martí-Carvajal AJ, Solà I, Lathyris D, Dayer M. Homocysteine-lowering interventions for preventing cardiovascular events. Cochrane Database Syst Rev. 2017:8:CD006612.

26. Katsiki N, Mikhailidis DP. Emerging vascular risk factors in women: any differences from men? Curr Med Chem. 2015;22:3565-79.

27. Piazzolla G, Castrovilli A, Liotino V, Vulpi MR, Fanelli M, Mazzocca A, et al. Metabolic syndrome and chronic obstructive pulmonary disease (COPD): the interplay among smoking, insulin resistance and vitamin D. PLoS ONE. 2017;12:e0186708.

28. Mursleen MT, Riaz S. Implication of homocysteine in diabetes and impact of folate and vitamine B12 in diabetic population. Diabetes Metab Syndr. 2017;11:S141-6.

29. Hayden MR, Tyagi SC. Homocysteine and reactive oxygen species in metabolic syndrome, type 2 diabetes mellitus and atheroscleropathy: the pleiotropic effects of folate supplementation. Nutr J. 2004;3:4.

30. American Diabetes Association. Standards of Medical Care in Diabetes-2018 Abridged for primary care providers. Clin Diabetes. 2018;36:14-37.

31. Xu C, Wu Y, Liu G, Liu X, Wang F, Yu J. Relationship between homocysteine level and diabetic retinopathy: a systematic review and meta-analysis. Diagn Pathol. 2014;9:167 
32. Pusparajah P, Lee LH, Abdul Kadir K. Molecular markers of diabetic retinopathy: potential screening tool of the future? Front Physiol. 2016;7:200

33. Mao S, Xiang W, Huang S, Zhang A. Association between homocysteine status and the risk of nephropathy in type 2 diabetes mellitus. Clin Chim Acta. 2014;431:206-10.

34. Wang H, Cui K, Xu K, Xu S. Association between plasma homocysteine and progression of early nephropathy in type 2 diabetic patients. Int Clin Exp Med. 2015:8:11174-80.

35. Tak YJ, Jeong DW, Kim YJ, Lee SY, Lee JG, Song SH, et al. Hyperhomocysteinaemia as a potential marker of early renal function decline in middleaged Asian people without chronic kidney disease. Int Urol Nephrol. 2016;48:239-48

36. Kernan WN, Obviagele B, Black HR, Bravata DM, Chimowitz MI, Ezekowitz MD, American Heart Association Stroke Council, Council on Cardiovascular and Stroke Nursing, Council on Clinical Cardiology, Council on Peripherl Vascular Disease, et al. Guidelines for the prevention of stroke in patients with stroke and transient ischemick attack: a guideline for healthcare professionals from the American Heart Association/American Stroke Association. Stroke. 2014;45:2160-236.

37. Towfighi A, Arshi B, Markovic D, Obviagele B. Homocysteine-lowering therapy and risk of recurrent stroke, myocardial infarction and death: the impact of age in the VISP trial. Cerebrovasc Dis. 2014;37:263-7.

38. Saposnik G, Ray JG, Sheridan P, McQueen M, Lonn E, Heart Outcomes Prevention Evaluation 2 Investigators. Homocysteine-lowering therapy and stroke risk, severity, and disability: additional findings from the HOPE 2 trial. Stroke. 2009;40(1365):72.
39. Huo Y, Li J, Qin X, Huang Y, Wang X, Gottesman RF, CSPPT Investigators, et al. Efficacy of folic acid therapy in primary prevention of stroke among adults with hypertension in China: the CSPPT randomized clinical trial. JAMA. 2015;313:1325-35.

40. Qin X, Li J, Spence JD, Zhang Y, Li Y, Wang X, et al. Folic acid therapy reduces the first stroke risk associated with hypercholesterolemia among hypertensive patients. Stroke. 2016;47:2805-12.

41. Yang HT, Lee M, Hong KS, Ovbiagele B, Saver JL. Efficacy of folic acid supplementation in cardiovascular disease prevention: an updated metaanalysis of randomized controlled trials. Eur J Intern Med. 2012;23:745-54.

42. Zeng $R, X u C H, X u Y N$, Wand $Y L$, Wang M. The effect of folate fortification on folic acid-based homocysteine-lowering intervention and stroke risk: a meta-analysis. Public Health Nutr. 2015;18:1514-21.

43. Zhang Q, Li S, Li L, Li Q, Ren K, Sun X, et al. Metformin treatment and homocysteine: a systematic review and meta-analysis of randomized controlled trials. Nutrients. 2016;8:798.

44. Garber AJ, Abrahamson MJ, Barzilay JI, Blonde L, Bloomgarden ZT, Bush MA, et al. Consensus statement by the American Association of Clinical Endocrinologists and American College of Endocrinology on the comprehensive type 2 diabetes management algorithm-2019 executive summary. Endocr Pract. 2019;25:69-100.

\section{Publisher's Note}

Springer Nature remains neutral with regard to jurisdictional claims in published maps and institutional affiliations.
Ready to submit your research? Choose BMC and benefit from:

- fast, convenient online submission

- thorough peer review by experienced researchers in your field

- rapid publication on acceptance

- support for research data, including large and complex data types

- gold Open Access which fosters wider collaboration and increased citations

- maximum visibility for your research: over 100M website views per year

At BMC, research is always in progress.

Learn more biomedcentral.com/submissions 